\title{
TEMPERATURA E SUBSTRATO PARA O TESTE DE GERMINAÇÃO DE SEMENTES DE BARBATIMÃO ((Stryphnodendron adstringens (Mart.) Coville (Leguminosae)). ${ }^{1}$
}

\author{
Chibele Chalita Martins², Carla Gomes Machado³ e João Nakagawa²
}

\begin{abstract}
RESUMO - As normas oficiais para análise de sementes não estabelecem critérios para a execução de testes de germinação da maioria das espécies florestais. Assim, o objetivo deste trabalho foi a determinação do substrato, da temperatura e da necessidade de superação da dormência das sementes para o teste de germinação de Stryphnodendron adstringens. Foram avaliados o tratamento de escarificação com ácido sulfúrico por 60 min, os substratos vermiculita, rolo de papel, areia e solo e as temperaturas constantes de $20,25,30$ e $35{ }^{\circ} \mathrm{C}$ e alternadas de $15-35^{\circ} \mathrm{C}$ e $20-30^{\circ} \mathrm{C}$, utilizando-se a primeira contagem de germinação (7 dias) e a porcentagem de plântulas normais, anormais, sementes mortas e dormentes ( $42^{\circ}$ dia após a semeadura) com quatro repetições de 50 sementes. As sementes de $S$. adstringens devem ser submetidas ao teste de germinação, após superação da dormência, em substrato papel e nas temperaturas constantes de 25 , 30 ou $35^{\circ} \mathrm{C}$ ou alternadas de $20-30{ }^{\circ} \mathrm{C}$.
\end{abstract}

Palavras-chave: Stryphnodendron adstringens, substrato, temperatura, dormência, planta medicinal e florestal.

\section{TEMPERATURE AND SUBSTRATE FOR GERMINATION TEST OF STRYPHNODENDRON ADSTRINGENS (MART) COVILLE (Leguminosae)}

\begin{abstract}
The official rules for seed testing don't establish criterions for accomplishment of germination test for greater number of forest species. Thus, the aim of this work was to define substrate, temperature and method for removing hard seededness for germination test of Stryphnodendron adstringens. They were studied: acid scarification with sulphuric acid for 60 minutes, substrates: vermiculita, rolled towel paper, sand and soil, and temperatures: constant, 20, 25, 30 and $35^{\circ} \mathrm{C}$, and alternating, $15-35^{\circ} \mathrm{C}$ and $20-30^{\circ} \mathrm{C}$. They were evalueted by first counting of the test (percentage of normal seedlings at 7 days after sowing), and the percentages of normal and abnormal seedlings, dormant and dead seeds (42 days after sowing), with four replications of 50 seeds. Seeds with hardness removed must be used to evaluate the germination of S. adstringens, in paper substrate and with constant temperature of 25,30 or $35^{\circ} \mathrm{C}$ or alternating of $20-30^{\circ} \mathrm{C}$.
\end{abstract}

Keywords:Stryphnodendron adstringens, substrate, temperature, dormancy, medicinal plant and forest seed.

\section{INTRODUÇÃO}

Stryphnodendron adstringens (Mart.) Coville (Leguminosae), conhecida como barbatimão, é uma árvore de Cerrado do Pará, Distrito Federal, Minas Gerais, São Paulo e Mato Grosso do Sul (LORENZI, 1992; FELFILI e SILVA JÚNIOR, 1993; FELFILI et al., 1999). A espécie apresenta a casca rica em tanino que tem ação antiinflamatória e cicatrizante, sendo utilizada triturada em aplicações na forma de emplastro sobre úlceras, impingens e hérnias e em solução de cozimento da casca para banhos e lavagens no combate de hemorragias

\footnotetext{
${ }^{1}$ Recebido em 11.05.2007 e aceito para publicação em 19.05.2008.

${ }^{2}$ Departamento de Produção Vegetal da Universidade Estadual Paulista Júlio de Mesquita Filho (UNESP), . E-mail : <cibele@fca.unesp.br>

${ }^{3}$ Programa de Pós-Graduação em Agronomia da UNESP.
} 
uterinas e afecções vaginais (LORENZI, 1992; LISBOA et al., 2006).

O extrativismo desordenado da casca que ocorre de forma independente do porte da planta é exercido pela população e estimulado por indústrias farmacêuticas e, aos poucos, vem provocando esgotamento deste recurso. Assim, é uma espécie que necessita de diretrizes para a sua conservação, desenvolvimento de técnicas de manejo sustentado e programas de domesticação e recomposição de matas nativas (BORGES FILHO e FELFILI, 2003).

O uso de sementes de qualidade é de grande importância para a propagação e produção de mudas e o teste de germinação é o principal parâmetro de avaliação da qualidade fisiológica das sementes. O resultado deste teste é utilizado para a determinação da taxa de semeadura, comparação do valor de lotes diferentes e comercialização, pois possibilita a obtenção de resultados comparáveis entre laboratórios. No entanto, para a maioria das sementes de espécies florestais nativas do Brasil os procedimentos do teste ainda não estão padronizados pelas Regras para Análise de Sementes (BRASIL, 1992), publicação que normatiza a metodologia deste teste, como a referente a recomendações de tratamentos de superação de dormência, substratos e temperaturas a serem utilizadas.

No teste de germinação, a temperatura atua sobre a velocidade de absorção de água e também sobre as reações bioquímicas que determinam todo o processo e, em conseqüência, afeta tanto a velocidade e uniformidade de germinação, como a germinação total (CARVALHO e NAKAGAWA, 2000). A germinação só ocorre dentro de determinados limites de temperatura, nos quais existe uma temperatura ótima, ou faixa de temperaturas, na qual o processo ocorre com a máxima eficiência (POPINIGIS, 1985; CARVALHO e NAKAGAWA, 2000).

Para as espécies florestais subtropicais e tropicais brasileiras a temperatura ótima de germinação situase entre 20 e $30^{\circ} \mathrm{C}$, uma vez que estas são temperaturas encontradas em suas regiões de origem, na época propícia para a germinação natural (BORGES e RENA, 1993). Assim, para sementes de espécies florestais da região amazônica como o pau-tanino, o jenipapo, a faveirapreta, a bacabinha e o jucá estas temperaturas são de $30^{\circ} \mathrm{C}$ (MIRANDA e FERRAZ, 1999; NASCIMENTO et al., 2000; NASCIMENTO et al., 2003; SILVA et al.,
2006; LIMA et al., 2006), de 30 a $35^{\circ} \mathrm{C}$ para o angelimpedra (VARELA et al., 2005) e 25 a $35^{\circ} \mathrm{C}$ para o paricá (RAMOS et al., 2006). Essas temperaturas ótimas de germinação, em sua maioria, são mais elevadas que as verificadas para espécies da mata atlântica, cerrados e matas da região sudeste e sul e do país, como o palmiteiro-jussara, o monjoleiro, o ipê-felpudo e o jacarandá-da-baía, que é de $25^{\circ} \mathrm{C}$ (BRASIL, 1992; ARAÚJO NETO et al., 2003; RAMOS et al., 2003; ANDRADE et al., 2006) e como a cataia, de $17^{\circ} \mathrm{C}$ (ABREU et al., 2005).

O substrato influencia diretamente a germinação, em função de sua estrutura, aeração, capacidade de retenção de água, propensão à infestação por patógenos, dentre outros, podendo favorecer ou prejudicar a germinação das sementes. Constitui o suporte físico no qual a semente é colocada e tem a função de manter as condições adequadas para a germinação e o desenvolvimento das plântulas (FIGLIOLIA et al., 1993). Portanto, o tipo de substrato utilizado deve ser adequado às exigências fisiológicas de germinação, tamanho e forma da semente (BRASIL, 1992). Assim, algumas espécies são mais exigentes, com desempenho germinativo superior em apenas um tipo de substrato, como a faveira-preta e jucá que devem ser semeadas entre areia (NASCIMENTO et al., 2003; LIMA et al., 2006); outras apresentam sementes mais adaptadas, germinam bem em vários substratos, como a bacabinha e o ipê-felpudo, que germinam tanto em areia como em vermiculita (MIRANDA e FERRAZ, 1999; RAMOS et al., 2003), o jacarandá-da-baía, em vermiculita e rolo de papel (ANDRADE et al., 2006), a cataia, em areia, ágar e sobre papel (ABREU et al., 2005) e a canafístula em papel, areia, xaxim e algodão (PEREZ et al., 2001).

Outras características da espécie podem dificultar ou impedir a germinação imediata da semente, a exemplo da impermeabilidade do tegumento à água, mecanismo de dormência comum no gênero Strypnodendron, a qual pode ser superada por escarificação mecânica ou ácida das sementes (VARELA et al., 1991; LEMUS FILHO et al., 1997).

Assim, o presente trabalho objetivou estudar a necessidade de superação da dormência das sementes, bem como a determinação do substrato e da temperatura para o teste de germinação de sementes de Stryphnodendron adstringens. 


\section{MATERIAL E MÉTODOS}

Frutos de Stryphnodendron adstringens foram colhidos em sete fragmentos de cerrado em 01 de agosto de 2006, localizados na região de Botucatu, Estado de São Paulo, em aproximadamente 95 plantas-mãe e transportados até o Laboratório de Análise de Sementes do Departamento de Produção Vegetal da Faculdade de Ciências Agronômicas da UNESP, onde as sementes foram extraídas manualmente das vagens e submetidas a limpeza para a retirada de sementes chochas, mal formadas e danificadas por fungos e insetos.

O trabalho foi realizado em duas etapas. Na primeira, estudou-se o desempenho germinativo de sementes submetidas ou não ao tratamento de superação de dormência de imersão em ácido sulfúrico por 60 minutos (MARTINS et al., 2007) nos seguintes substratos: rolo de papel, sobre solo, sobre areia e sobre vermiculita. A granulometria do solo, areia média e vermiculita fina utilizados no experimento situou-se, respectivamente, entre 0,062 e $0,031 \mathrm{~mm} ; 0,500$ e 0,250 mm e ainda 2,000 e $1,000 \mathrm{~mm}$.

O umedecimento dos substratos, areia e solo foi realizado com, respectivamente, $60 \%$ e $50 \%$ da capacidade de campo em água, e dos substratos vermiculita e papel com, respectivamente, 1,3 e 2,5 vezes o peso do substrato em água (BRASIL, 1992). Para que a distância entre as sementes postas para germinar fosse a mesma nos quatro substratos avaliados, o teste em areia, solo e vermiculita foi conduzido em caixas plásticas de 30,2 x 20,8 x 6,3cm, similar em comprimento e largura às dimensões do papel utilizado em rotina nos testes de germinação em laboratórios de análise de sementes.

O efeito dos tratamentos sobre o desempenho germinativo das sementes foi avaliado pelos testes de germinação e primeira contagem de germinação utilizando-se quatro subamostras de 50 sementes, colocadas para germinar nos diferentes substratos, na temperatura de $25^{\circ} \mathrm{C}$ e $8 \mathrm{~h}$ de luz. A avaliação do teste da primeira contagem foi realizada aos sete dias após a semeadura, contabilizando-se a porcentagem de plântulas normais; enquanto a avaliação do teste de germinação foi efetuada semanalmente, do sétimo ao $42^{\circ}$ dia após a semeadura, quando foram calculadas as porcentagens de germinação (plântulas normais), plântulas anormais, sementes dormentes e mortas. Nas câmaras de germinação, os rolos de papel e as caixas plásticas com substrato permaneceram acondicionados dentro de sacos plásticos de 0,033 mm de espessura fechados, para evitar a desidratação (COIMBRA et al., 2007).

Os tratamentos mais favoráveis à germinação, selecionados na primeira etapa, foram utilizados na segunda etapa do trabalho, quando foram avaliadas as temperaturas constantes de $20,25,30$ e $35^{\circ} \mathrm{C}$ e alternadas de $15-35^{\circ} \mathrm{C}$ e $20-30{ }^{\circ} \mathrm{C}$ no desempenho germinativo das sementes, pelo teste de germinação e da primeira contagem de germinação realizados com a mesma metodologia adotada na primeira etapa.

O experimento foi instalado em um delineamento estatístico inteiramente casualizado e com quatro repetições. Na primeira etapa da pesquisa os tratamentos foram dispostos em um esquema fatorial $4 \times 2$ (substrato x superação da dormência) e na segunda etapa, utilizouse um fatorial $6 \times 2$, sendo seis regimes de temperatura avaliados e dois substratos que foram selecionados da primeira etapa do trabalho. Realizou-se a transformação dos dados em $(x+0,5) 1 / 2$, antes das análises, para parâmetros com valores iguais a zero e em arco-seno (x/100)1/2 para valores menores que $15 \%$ ou maiores que $85 \%$, de acordo com as recomendações de Banzatto e Kronka (2006). As médias apresentadas nos quadros são de dados não transformados.

\section{RESULTADO E DISCUSSÃO}

O tratamento de superação de dormência foi eficiente, tornando zero a porcentagem de sementes dormentes, em todos os substratos avaliados. Adicionalmente, o tratamento ocasionou aumento na porcentagem de germinação, de valores médios de $7,75 \%$ para $83 \%$, e maior velocidade de germinação, verificada pelo teste da primeira contagem (Quadro 1). Embora o tratamento tenha aumentado significativamente a ocorrência de sementes mortas nos substratos areia, papel e terra, os efeitos favoráveis à germinação foram numericamente maiores e mais expressivos, validando a utilização do tratamento de superação de dormência antes da instalação do teste de germinação de sementes de $S$. adstringens.

De forma similar, a eficiência do tratamento com ácido sulfúrico na superação da dormência foi constatada para sementes de Stryphnodendron pulcherrimum (VARELA et al., 1991) e Tachigalia multijuga (BORGES et al., 2004) e Zizyphus joazeiro (ALVES et al., 2006), entre outras espécies.

R. Árvore, Viçosa-MG, v.32, n.4, p.633-639, 2008 
Quadro 1 - Primeira contagem de germinação, germinação (plântulas normais, anormais), sementes mortas e dormentes de barbatimão (S. adstringens) submetidas ou não a tratamento de superação de dormência (D) com ácido sulfúrico e colocadas para germinar em diferentes substratos (S)

Table 1 - Means data of first counting of germination test, germination, abnormal seedlings, dead and dormant seeds of S. adstringens after submited or not to treatment with sulphuric acid for hard seededness removing (D) and put to germinate in different subtrates $(S)$

\begin{tabular}{|c|c|c|c|c|c|c|c|c|c|}
\hline \multirow{2}{*}{$\begin{array}{c}\text { Variáveis } \\
\text { avaliadas (\%) }\end{array}$} & \multirow[t]{2}{*}{ Substrato (S) } & \multicolumn{4}{|c|}{ Tratamento de superação de dormência (D) } & \multirow[t]{2}{*}{ FS } & \multirow[t]{2}{*}{ FD } & \multirow[t]{2}{*}{ F SxD } & \multirow[t]{2}{*}{ C.V.\% } \\
\hline & & \multicolumn{2}{|c|}{ Sem } & \multicolumn{2}{|c|}{ Com } & & & & \\
\hline \multirow{4}{*}{$\begin{array}{l}\text { Primeira contagem de } \\
\text { germinação }\end{array}$} & Areia & 1 & a B & 63 & $\mathrm{bA}$ & \multirow{4}{*}{$15,71^{* *}$} & \multirow{4}{*}{$1641,76^{* *}$} & \multirow{4}{*}{$15,36 * *$} & \multirow{4}{*}{13,7} \\
\hline & Terra & 1 & a B & 59 & bA & & & & \\
\hline & Vermiculita & 1 & a B & 73 & $\mathrm{aA}$ & & & & \\
\hline & Papel & 1 & a B & 89 & $\mathrm{aA}$ & & & & \\
\hline \multirow{4}{*}{ Germinação } & Areia & 10 & a B & 69 & $\mathrm{bA}$ & \multirow{4}{*}{$9,60 * *$} & \multirow{4}{*}{$3723,33 * *$} & \multirow{4}{*}{$21,93 * *$} & \multirow{4}{*}{7,7} \\
\hline & Terra & 9 & a B & 83 & $\mathrm{aA}$ & & & & \\
\hline & Vermiculita & 6 & a B & 89 & $\mathrm{aA}$ & & & & \\
\hline & Papel & 6 & a B & 89 & $\mathrm{aA}$ & & & & \\
\hline \multirow{4}{*}{ Plântulas anormais } & Areia & 0 & $\mathrm{bA}$ & 0 & $\mathrm{bA}$ & \multirow{4}{*}{$5,07 * *$} & \multirow{4}{*}{$0,22 \mathrm{~ns}$} & \multirow{4}{*}{$5,72 * *$} & \multirow{4}{*}{35,5} \\
\hline & Terra & 0 & bB & 2 & $\mathrm{aA}$ & & & & \\
\hline & Vermiculita & 2 & $\mathrm{aA}$ & 1 & $\mathrm{abB}$ & & & & \\
\hline & Papel & 0 & bA & 0 & bA & & & & \\
\hline \multirow{4}{*}{ Sementes mortas } & Areia & 3 & $\mathrm{bB}$ & 31 & $\mathrm{aA}$ & \multirow{4}{*}{2,63 ns } & \multirow{4}{*}{$72,18 * *$} & \multirow{4}{*}{$15,54 * *$} & \multirow{4}{*}{18,5} \\
\hline & Terra & 5 & $\mathrm{abB}$ & 15 & bA & & & & \\
\hline & Vermiculita & 9 & $\mathrm{aA}$ & 10 & $\mathrm{bA}$ & & & & \\
\hline & Papel & 5 & abB & 11 & bA & & & & \\
\hline \multirow{4}{*}{ Sementes dormentes } & Areia & 88 & $\overline{a b A}$ & 0 & $\overline{a B}$ & \multirow{4}{*}{1,65 ns } & \multirow{4}{*}{$8005,94 * *$} & \multirow{4}{*}{1,65 ns } & \\
\hline & Terra & 87 & abA & 0 & a B & & & & 6,3 \\
\hline & Vermiculita & 84 & bA & 0 & a B & & & & \\
\hline & Papel & 90 & $\mathrm{aA}$ & 0 & a B & & & & \\
\hline
\end{tabular}

$\mathrm{ns}, *$ e **: não-significativo e significativo a $5 \%$ e $1 \%$ pelo teste $\mathrm{F}$, respectivamente.

Médias seguidas por letras iguais, minúsculas na coluna e maiúsculas na linha, não diferem pelo teste de Tukey a 5\% de probabilidade.

As sementes submetidas ao tratamento de superação de dormência e semeadas nos substratos terra, vermiculita e papel indicaram os melhores resultados de germinação e de sementes mortas que foram, respectivamente, superiores e inferiores aos do substrato areia. Não obstante, o substrato terra aumentou a incidência de plântulas anormais, embora em valores de $2 \%$, numericamente pequeno. Com base no teste da primeira contagem, os substratos vermiculita e papel favorecem ao processo de germinação fazendo com que o mesmo ocorra com maior velocidade. Por isso, tais substratos foram selecionados para serem mais bem avaliados na segunda etapa da pesquisa, considerando que o teste de germinação deve ser conduzido nas condições ótimas de cada espécie para garantir que o lote possa expressar seu potencial máximo de germinação (BRASIL, 1992). A semeadura em rolo de papel e sobre vermiculita podem ter apresentado melhores resultados devido a maior capacidade de drenagem e aeração desses substratos em comparação com os demais; pois, entre os minerais, a vermiculita apresentou a maior granulometria e o rolo de papel oferece menor superfície de contato à semente que os demais substratos.

Considerando que não houve efeito da interação entre as temperaturas e os substratos utilizados (Quadro 2 ), dessa forma os tratamentos foram avaliados com base nos fatores principais. As diversas temperaturas não afetaram a porcentagem de germinação, plântulas anormais, sementes mortas e sementes dormentes. $\mathrm{O}$ mesmo foi verificado para os substratos testados, com exceção do substrato papel, no qual as sementes produziram uma maior porcentagem de plântulas anormais.

Somente no teste da primeira contagem foi possível identificar o substrato e as temperaturas mais favoráveis ao processo de germinação das sementes de $S$. adstringens, que foi o substrato papel e os regimes de temperaturas constantes de 25,30 e $35^{\circ} \mathrm{C}$ e alternadas de $20-30^{\circ} \mathrm{C}$. Essas condições permitem que as sementes 
germinem mais rapidamente garantindo a expressão do seu potencial máximo de germinação, como recomendam as Regras para Análise de Sementes para este teste (BRASIL, 1992). Valores estatisticamente inferiores foram obtidos no teste da primeira contagem de germinação, nas temperaturas relativamente mais baixas, de $20^{\circ} \mathrm{C}$ e $15-35^{\circ} \mathrm{C}$.

De acordo com tais resultados, as sementes de S. adstringens são exigentes quanto ao substrato desempenhando-se melhor em um deles, comportamento esse, similar ao de faveira-preta e jucá (NASCIMENTO et al., 2003; LIMA et al., 2006). No entanto, são mais adaptadas quanto à temperatura de germinação, desempenhando-se bem numa faixa bastante ampla, a semelhança do constatado com sementes de paricá (RAMOS et al., 2006).

\section{CONCLUSÕES}

As sementes de $S$. adstringens devem ser submetidas ao teste de germinação, após superação da dormência, em substrato papel e nas temperaturas constantes de 25,30 ou $35^{\circ} \mathrm{C}$ ou alternadas de 20 $30{ }^{\circ} \mathrm{C}$, por serem as condições mais favoráveis para o desempenho germinativo das sementes.

\section{REFERÊNCIAS}

ALVES, E.U.; BRUNO, R.L.A.; OLIVEIRA, A.P.; ALVES. A.U.; ALVES. A.U. Ácido sulfúrico para superação da dormência de unidade de dispersão de juazeiro (Zizyphus joazeiro Mart.). Revista Árvore, Viçosa, v.30, n.2, p.187-195, 2006.

ABREU, D.C.A.; NOGUEIRA, A.C.; MEDEIROS, A.C. de S. Efeito do substrato e da temperatura na germinação de sementes de cataia (Drimys brasiliensis Miers. WINTERACEAE). Revista Brasileira Sementes, Pelotas, v. 27, n.2, p. 149-157, 2005.

ANDRADE, A.C.S.; PEREIRA, T. S.; FERNANDES, M. J.; CRUZ, A. P. M.; CARVALHO, A. S. R. Germinação de sementes de Dalbergia nigra (Vell.) Fr. All. Ex Benth: substrato, temperatura e desenvolvimento pós-seminal. Pesquisa Agropecuária Brasileira, Brasília, v. 41, n. 3, p. 517-523, 2006.

ARAUJO NETO, J.C.A.; AGUIAR, I.B.;

FERREIRA, V.M. Efeito da temperatura e da luz na germinação de sementes de Acacia polyphylla dc.

Revista Brasileira Botânica, São

Quadro 2 - Temperatura e substrato sobre a primeira contagem, germinação, plântulas anormais, sementes mortas e dormentes em sementes de barbatimão (S. adstringens)

Table 2 - Means data of first counting of germination test, germination abnormal seedlings, dead and dormant seeds of S. adstringens after put to germinate at differents temperatures $(T)$ and two substrates $(S)$

\begin{tabular}{|c|c|c|c|c|c|c|c|c|c|c|}
\hline $\begin{array}{r}\text { Tratamentos } \\
\text { Temperatura (T) }\end{array}$ & \multicolumn{2}{|c|}{$\begin{array}{c}\text { Primeira contagem } \\
(\%)\end{array}$} & \multicolumn{2}{|c|}{ Germinação (\%) } & \multicolumn{2}{|c|}{$\begin{array}{c}\text { Plântulas } \\
\text { anormais (\%) }\end{array}$} & \multicolumn{2}{|c|}{ Sementes mortas (\%) } & \multicolumn{2}{|c|}{$\begin{array}{c}\text { Sementes dormentes } \\
(\%)\end{array}$} \\
\hline & & & & & & & & & & \\
\hline $20^{\circ} \mathrm{C}$ & 66,5 & $\mathrm{~b}$ & 85,8 & $\mathrm{a}$ & 1,3 & $\mathrm{a}$ & 13,0 & $\mathrm{a}$ & 0,0 & $\mathrm{a}$ \\
\hline $25^{\circ} \mathrm{C}$ & 78,0 & a & 86,3 & $\mathrm{a}$ & 0,8 & a & 10,0 & $\mathrm{a}$ & 0,0 & $\mathrm{a}$ \\
\hline $30^{\circ} \mathrm{C}$ & 77,5 & a & 86,3 & a & 1,0 & a & 12,5 & $\mathrm{a}$ & 0,0 & $\mathrm{a}$ \\
\hline $35^{\circ} \mathrm{C}$ & 79,3 & a & 86,8 & $\mathrm{a}$ & 1,5 & a & 12,0 & $\mathrm{a}$ & 0,0 & $\mathrm{a}$ \\
\hline $20-30{ }^{\circ} \mathrm{C}$ & 77,0 & $\mathrm{a}$ & 87,5 & $\mathrm{a}$ & 1,5 & a & 11,3 & $\mathrm{a}$ & 0,0 & $\mathrm{a}$ \\
\hline $15-35^{\circ} \mathrm{C}$ & 75,8 & $a b$ & 89,3 & $\mathrm{a}$ & 2,0 & $\mathrm{a}$ & 11,8 & $\mathrm{a}$ & 0,0 & $\mathrm{a}$ \\
\hline \multicolumn{11}{|l|}{ Substrato (S) } \\
\hline Vermiculita & 72,9 & b & 86,8 & a & 0,6 & a & 12,6 & a & 0,1 & a \\
\hline Papel & 78,4 & $\mathrm{a}$ & 87,2 & a & 2,1 & $\mathrm{~b}$ & 10,8 & $\mathrm{a}$ & 0,0 & $\mathrm{a}$ \\
\hline F T & 3,86 & $* *$ & 0,67 & ns & 0,57 & ns & 0,40 & $\overline{\mathrm{ns}}$ & 1,00 & $\overline{\mathrm{ns}}$ \\
\hline F S & 7,66 & $* *$ & 0,11 & ns & 6,20 & $*$ & 1,79 & ns & 1,00 & ns \\
\hline F TxS & 0,17 & ns & 2,23 & ns & 1,32 & ns & 2,38 & ns & 1,00 & ns \\
\hline C.V. \% & \multicolumn{2}{|c|}{4,5} & \multicolumn{2}{|r|}{2,5} & \multicolumn{2}{|c|}{142,2} & \multicolumn{2}{|c|}{17,6} & \multicolumn{2}{|c|}{692,8} \\
\hline
\end{tabular}

$\mathrm{ns}, *$ e **: não-significativo e significativo a $5 \%$ e $1 \%$ pelo teste $\mathrm{F}$, respectivamente.

Médias seguidas por letras iguais na coluna não diferem entre si, pelo teste de Tukey a 5\% de probabilidade. 
Paulo,v.26, n. 2, p. 249-256, 2003.

BANZATTO, D.A.; KRONKA, S.N. Experimentação agrícola. 4.ed. Jaboticabal: FUNEP, 2006. 237p.

BORGES FILHO, H. C.; FELFILI, J. M. Avaliação dos níveis de extrativismo da casca de barbatimão (Stryphnodendron adstringens (Mart.) Coville) no Distrito Federal, Brasil. Revista Árvore, Viçosa, v. 27, n. 5, 2003.

BORGES, E.E.L.; RENA, A.B. Germinação de sementes. In: AGUIAR, I.B.; PIÑA RODRIGUES, F.C.M.; FIGLIOLIA, M.B. Sementes florestais tropicais. Brasília: ABRATES, 1993. p. 83-136.

BORGES, E.E.L.; RIBEIRO JUNIOR, J.I.; REZENDE, S. T.; PEREZ S.C.J.G.A. Alterações fisiológicas em sementes de Tachigalia multijuga (Benth.) (mamoneira) relacionadas aos métodos para a superação da dormência. Revista Árvore, Viçosa, v.28, n.3, p.317-325, 2004.

BRASIL. MINISTÉRIO DAAGRICULTURAE REFORMA AGRARIA. Regras para análise de sementes. Brasília: SNDA/DNDV/CLAV, 1992. 365p.

CARVALHO, N.M.; NAKAGAWA, J. Sementes: ciência, tecnologia e produção. 4.ed. Jaboticabal: FUNEP, 2000. 588p.

COIMBRA, R.A., TOMAZ, C.A., MARTINS, C.C.; NAKAGAWA, J. Teste de germinação com acondicionamento dos rolos de papel em sacos plásticos visando a otimização dos resultados. Revista Brasileira Sementes, Brasília, v. 29, n.1, p.92-97, 2007.

FELFILI J. M.; SILVA JUNIOR, M.C.; DIAS, B.J. E REZENDE, A.V. Estudo fenológico de Stryphnodendron adstringens (Mart.) Coville no cerrado sensu stricto da Fazenda Água Limpa no Distrito Federal, Brasil. Revista Brasileira Botânica, São Paulo, v.22, n.1, p.83-90, 1999.

FELFILI, J.M. E SILVA JÚNIOR, M.C. A comparative study of cerrado (sensu stricto)

R. Árvore, Viçosa-MG, v.32, n.4, p.633-639, 2008 vegetation in central Brazil. Journal of Tropical Ecology, Cambridge, v. 9, n.3, p.277-289, 1993.

FIGLIOLIA, M.B.; OLIVEIRA, E.C.; PIÑA RODRIGUES, F.C.M. Análise de sementes. In: AGUIAR, I.B.; PIÑA RODRIGUES, F.C.M.; FIGLIOLIA, M.B. (eds.). Sementes florestais tropicais. Brasília: ABRATES, 1993. p. 137-174.

LEMUS FILHO, J.P.; GUERRA, S.T.M.; LOVATO, M.B. E SCOTTI, M.R.M.M.L. Germinação de sementes de Senna macranthera, Senna multijuga e Stryphnodendron polyphyllum. Pesquisa Agropecuária Brasileira, Brasília, v.32, n.4, p.357-361, 1997.

LIMA, J.D.; ALMEIDA, C.C.; DANTAS, V.A.V.; SILVA, B.M.S., MORAES, W.S. Efeito da temperatura e do substrato na germinação de sementes de Caesalpinia ferrea Mart. ex Tul. (Leguminosae, Caesalpinoideae). Revista Árvore, Viçosa, v. 30, n. 4, 2006.

LISBOA, M.S.; FERREIRA, S.M.;SILVA, M.S. Uso de plantas medicinais para tratar úlceras e gastrites. Sitientibus, Serie Ciências Biológicas, v. 6, n. Especial - Etnobiologia, p. 13-20, 2006

LORENZI, H. Árvores brasileiras: manual de identificação e cultivo de plantas arbóreas nativas do Brasil. Nova Odessa: Instituto Plantarum, v.1., 1992. 373 p.

MARTINS, C. C., CAMARA, A. T. R. da, MACHADO, C. G., NAKAGAWA, J. Germination of seed submitted to treatments for breaking dormancy In: 28 ISTA Congress e XV Congresso Brasileiro de Sementes, 2007, Foz do Iguaçu-PR.

Seed Symposium Abstracts “Diversity in Seed Technology”., 2007. p.83 - 83 .

MIRANDA, P.R.M. E FERRAZ, I.D.K. Efeito da temperatura na germinação de sementes e morfologia da plântula de Maquira sclerophylla (Ducke). Revista Brasileira Botânica, São Paulo, v.22, n.2, p.303-307, 1999.

NASCIMENTO, W. M. O. do ; CARVALHO, J. E. 
U. E CARVALHO, N. M. . Germinação de sementes de jenipapo (Genipa americana L.) submetidas a diferentes temperaturas e substratos. Revista Brasileira Fruticultura, Jaboticabal, v. 22, n. 3, p. 471-473, 2000.

NASCIMENTO, W. M. O. do ; RAMOS, N.P ; CARPI, V.A.F. ; SCARPARE FILHO, J. A. ;

CRUZ, E.D. Temperatura e substrato para germinação de sementes de Parkia platycephala Benth. (LeguminosaeCaesalpinoideae). Revista de

Agricultura Tropical, Cuiabá, v. 7, n. 1, p. 119-129, 2003.

PEREZ, S. C. J. G.A.; FANTI, S. C.; CASALI, C. A. Influência da luz na germinação de sementes de canafístula submetidas ao estresse hídrico. Bragantia, Campinas, v. 60, n. 3, p. 155-166, 2001.

POPINIGIS, F. Fisiologia de Sementes. 2. ed. Brasília: ABRATES, 1985. 298 p.
RAMOS, M.B.P. ; VARELA, V.P.; MELO, M.F.F. Influência da temperatura e da água sobre a germinação de sementes de Paricá (Schizolobium amazonicum Huber ex Ducke Leguminosae-Caesalpinioideae). Revista Brasileira Sementes, Pelotas, v.28, n.1, p. 163-168, 2006.

RAMOS, N. P. ; MENDOÇA, E. A. F. ; PAULA, R. C. . Germinação de sementes de Zeyhera tuberculosa (Vell.) Bur. (Ipê-felpudo). Revista Agricultura Tropical, Cuiabá, v.7, n. 1, p.41-52, 2003.

SILVA, B.M.S.; CESARINO, F.; LIMA, J.D.; PANTOJA, T.F.; MORO, F.V. Germinação de sementes e emergência de plântulas de Oenocarpus minor Mart. (Arecaceae). Revista Brasileira Fruticultura, Jaboticabal, v.28, n.2, p.289-292, 2006.

VARELA, V.P. ; BROCKI, E. E SÁ, S.T.V. Tratamentos pré-germinativos de sementes de espécies florestais da Amazônia: IV. Faveira camuzê - Stryphnodendron pulcherrimum (Willd.) Hochr Leguminosae. Revista Brasileira Sementes, Brasília, v.13, n.2, p. 87-90, 1991.

VARELA, V.P.; RAMOS, M.B.P.; MELO, M.F.F. Umedecimento do substrato e temperatura na germinação de sementes de angelim-pedra (Dinizia excelsa Ducke). Revista Brasileira Sementes, Pelotas, v.27, n.2, p.130-135, 2005. 